\title{
A REMARK ON MINIMAL NODAL SOLUTIONS OF AN ELLIPTIC PROBLEM IN A BALL
}

\author{
Olaf TORnÉ
}

\author{
(Submitted by Michel Willem)
}

\begin{abstract}
Consider the equation $-\Delta u=u_{+}^{p-1}-u_{-}^{q-1}$ in the unit ball $B$ with a homogeneous Dirichlet boundary condition. We assume $2<p, q<$ $2^{*}$. Let $\varphi(u)=(1 / 2) \int_{B}|\nabla u|^{2} d x-(1 / p) \int_{B} u_{+}^{p} d x-(1 / q) \int_{B} u_{-}^{q} d x$ be the functional associated to this equation. The nodal Nehari set is defined by $\mathcal{M}=\left\{u \in H_{0}^{1}(B): u_{+} \neq 0, u_{-} \neq 0,\left\langle\varphi^{\prime}\left(u_{+}\right), u_{+}\right\rangle=\left\langle\varphi^{\prime}\left(u_{-}\right), u_{-}\right\rangle=0\right\}$. Now let $\mathcal{M}_{\text {rad }}$ denote the subset of $\mathcal{M}$ consisting of radial functions and let $\beta_{\text {rad }}$ be the infimum of $\varphi$ restricted to $\mathcal{M}_{\text {rad }}$. Furthermore fix two disjoint half balls $B^{+}$and $B^{-}$and denote by $\mathcal{M}_{h}$ the subset of $\mathcal{M}$ consisting of functions which are positive in $B^{+}$and negative in $B^{-}$. We denote by $\beta_{h}$ the infimum of $\varphi$ restricted to $\mathcal{M}_{h}$. In this note we are interested in obtaining inequalities between $\beta_{\text {rad }}$ and $\beta_{h}$. This problem is related to the study of symmetry properties of least energy nodal solutions of the equation under consideration. We also consider the case of the homogeneous Neumann boundary condition.
\end{abstract}

\section{Introduction}

Let $B$ denote the open unit ball in $\mathbb{R}^{N}$ with $N \geq 2$. Consider the problem

$$
\begin{cases}-\Delta u=u_{+}^{p-1}-u_{-}^{q-1} & \text { in } B, \\ u=0 & \text { on } \partial B,\end{cases}
$$

where $2<p, q<2^{*}$ and $2^{*}$ is the critical Sobolev exponent. When $q=p$ the above equation reduces to $-\Delta u=|u|^{p-2} u$ which is the model equation in

2000 Mathematics Subject Classification. Primary 35J60; Secondary 35J25.

Key words and phrases. Nodal solutions, Symmetry, Elliptic semilinear equations. 
many studies on semilinear elliptic problems. We are interested in minimal sign changing solutions of (1.1).

Least energy, or minimal, sign changing solutions have been obtained in [1] for quite general equations $-\Delta u=f(x, u)$ in smooth bounded domains (see also [2] and references therein). In the special case of problem (1.1) these nodal solutions are obtained in the following way. Let the functional

$$
\varphi(u)=\frac{1}{2} \int_{B}|\nabla u|^{2} d x-\frac{1}{p} \int_{B} u_{+}^{p} d x-\frac{1}{q} \int_{B} u_{-}^{q} d x
$$

be defined over $H_{0}^{1}(B)$. A function $u$ is a solution of (1.1) if and only if it is a critical point of $\varphi$. Define the nodal Nehari set by

$$
\mathcal{M}=\left\{u \in H_{0}^{1}(B): u_{+} \neq 0, u_{-} \neq 0,\left\langle\varphi^{\prime}\left(u_{+}\right), u_{+}\right\rangle=\left\langle\varphi^{\prime}\left(u_{-}\right), u_{-}\right\rangle=0\right\} .
$$

It is shown in [1] and [2] that

$$
\beta=\inf _{u \in \mathcal{M}} \varphi(u)
$$

is achieved and, moreover, any minimizer $u \in \mathcal{M}$ is a critical point of $\varphi$ having exactly two nodal domains. Such a function $u$ is called a least energy nodal solution of (1.1).

The symmetry properties of least energy nodal solutions have been studied in [2]. The authors show that minimal nodal solutions of $-\Delta u=f(x, u)$ in a radial domain with a quite general nonlinearity $f(x, u)$ are foliated Schwartz symmetric. An interesting question is to obtain further information about the shape of the nodal domains of least energy sign changing solutions. Let $\mathcal{A}$ denote the set of pairs $(\omega, \widetilde{\omega})$ where $\omega$ and $\widetilde{\omega}$ are disjoint nonempty open subsets of $B$. Then we have the following characterization

$$
\beta=\min _{(\omega, \widetilde{\omega}) \in \mathcal{A}} \frac{p-2}{2 p} S_{p}(\omega)^{p /(p-2)}+\frac{q-2}{2 q} S_{q}(\widetilde{\omega})^{q /(q-2)}
$$

where $S_{p}(\omega)$, given by equation (1.5) below, is the best Sobolev constant for the injection $H_{0}^{1}(\omega) \hookrightarrow L^{p}(\omega)$. Moreover the minimum in (1.4) is achieved if and only if $\omega$ and $\widetilde{\omega}$ are the nodal domains of some minimizer $u$ of $\beta$. This formula is presumably well known but we recall the proof further on for the reader's convenience.

The problem of describing the shape of the nodal domains of least energy sign changing solutions is thus equivalent to finding a pair of domains $\omega$ and $\widetilde{\omega}$ which minimize (1.4). Two natural structures have been suggested. First consider

$$
\beta_{\mathrm{rad}}=\inf _{u \in \mathcal{M}_{\mathrm{rad}}} \varphi(u)
$$

where $\mathcal{M}_{\text {rad }}$ is the subset of $\mathcal{M}$ consisting of radial functions. One may show that $\beta_{\mathrm{rad}}$ is achieved by a radial sign changing solution $v$ of (1.1) which has exactly two nodal domains (see [2]). Clearly the nodal domains of $v$ are a ball 
and an annulus. Next let $B^{ \pm}=\left\{x \in B ; \pm x_{N}>0\right\}$ be half balls and denote by $\mathcal{M}_{h}$ the subset of $\mathcal{M}$ consisting of functions which are positive in $B^{+}$and negative in $B^{-}$. The infimum of $\varphi$ over $\mathcal{M}_{h}$, denoted by $\beta_{h}$, is achieved and we have

$$
\beta_{h}=\frac{p-2}{2 p} S_{p}\left(B^{+}\right)^{p /(p-2)}+\frac{q-2}{2 q} S_{q}\left(B^{-}\right)^{q /(q-2)} .
$$

When $q=p$ and $B$ is replaced by an annulus it is shown in [2] that $\beta \leq$ $\beta_{h}<\beta_{\text {rad }}$ if $p$ is sufficiently near to $2^{*}$. We are interested in studying this kind of inequality in the case of problem (1.1).

THEOREM 1.1. Let $\beta_{h}$ and $\beta_{\mathrm{rad}}$ be as above.

(a) If $p$ is sufficiently near to 2 and if $q$ is sufficiently near to $p$ then $\beta_{h}<$ $\beta_{\mathrm{rad}}$. In particular if $q=p$ is near to 2 then the conclusion holds.

(b) Let $p$ be fixed with $2<p<2^{*}$. If $q$ is sufficiently near to 2 or if $q$ is sufficiently near to $2^{*}$ then $\beta_{\mathrm{rad}}<\beta_{h}$.

A statement similar to (a) holds when $q$ is fixed and $p$ varies.

A similar result to Theorem 1.1 holds when the Dirichlet boundary condition appearing in equation (1.1) is replaced by a homogeneous Neumann condition $\partial u / \partial \nu=0$. The Neumann problem is considered in greater detail in Section 3 .

\section{Proof of Theorem 1.1}

In order to prove Theorem 1.1 we will recall some characterizations of the minima under consideration. Let us first introduce some notations. Let $\omega \subset B$ be a nonempty open subset of $B$ and denote by

$$
S_{p}(\omega)=\inf _{u \in H_{0}^{1}(\omega)} \frac{\int_{\omega}|\nabla u|^{2} d x}{\left(\int_{\omega}|u|^{p} d x\right)^{2 / p}}
$$

the best Sobolev constant for the injection

$$
H_{0}^{1}(\omega) \hookrightarrow L^{p}(\omega) \text { with } 2 \leq p \leq 2^{*}
$$

When $\omega$ is a radial domain we denote by $S_{p}^{\mathrm{rad}}(\omega)$ the infimum (2.1) taken over radial functions. Note that if $\omega$ is a ball then $S_{p}^{\operatorname{rad}}(\omega)=S_{p}(\omega)$ whereas if $\omega$ is an annulus this is not always the case. Denote by $\mathcal{A}$ be the set of pairs $(\omega, \widetilde{\omega})$ where $\omega$ and $\widetilde{\omega}$ are disjoint nonempty open subsets of $B$. Let $r \in] 0,1[$. We denote by $B_{r}$ the open ball of radius $r$ centered at the origin and $A_{r}=B \backslash \bar{B}_{r}$ the annulus of unit outer radius and of inner radius $r$. 
Proposition 2.1. Let $2<p, q<2^{*}$. Then we have the following characterizations.

$$
\begin{aligned}
\beta= & \min _{(\omega, \widetilde{\omega}) \in \mathcal{A}} \frac{p-2}{2 p} S_{p}(\omega)^{p /(p-2)}+\frac{q-2}{2 q} S_{q}(\widetilde{\omega})^{q /(q-2)}, \\
\beta_{h}= & \frac{p-2}{2 p} S_{p}\left(B^{+}\right)^{p /(p-2)}+\frac{q-2}{2 q} S_{q}\left(B^{-}\right)^{q /(q-2)}, \\
\beta_{\mathrm{rad}}= & \min _{0<r<1} \min \left\{\frac{p-2}{2 p} S_{p}\left(B_{r}\right)^{p /(p-2)}+\frac{q-2}{2 q} S_{q}^{\mathrm{rad}}\left(A_{r}\right)^{q /(q-2)} ;\right. \\
& \left.\frac{p-2}{2 p} S_{p}^{\mathrm{rad}}\left(A_{r}\right)^{p /(p-2)}+\frac{q-2}{2 q} S_{q}\left(B_{r}\right)^{q /(q-2)}\right\} .
\end{aligned}
$$

Moreover, the minimum in (2.2) is achieved if and only if $\omega$ and $\widetilde{\omega}$ are the nodal domains of some minimizer $u$ for $\beta$. Likewise the minima in (2.4) are achieved only by the nodal domains of some minimizer for $\beta_{\mathrm{rad}}$.

Proof. We will just prove (2.2) since (2.3) and (2.4) follow in the same way. It is straightforward to check that $\beta$ defined by (1.3) has the characterization

$$
\beta=\min _{(\omega, \widetilde{\omega}) \in \mathcal{A}} c(\omega)+c(\widetilde{\omega})
$$

where

$$
c(\omega)=\inf _{u \in \mathcal{N}(\omega)} \varphi(u) \quad \text { and } \quad \mathcal{N}(\omega)=\left\{u \in H_{0}^{1}(\omega): u>0,\left\langle\varphi^{\prime}(u), u\right\rangle=0\right\}
$$

for any nonempty open subset $\omega \subset B$. Moreover, the minimum in (2.5) is reached if and only if $\omega$ and $\widetilde{\omega}$ are the nodal domains of some minimizer $u$ of $\beta$. Notice that $\mathcal{N}(\omega)$ is a Nehari manifold. It is well known that

$$
c(\omega)=\frac{p-2}{2 p} S_{p}(\omega)^{p /(p-2)} \quad \text { and } \quad c(\widetilde{\omega})=\frac{q-2}{2 q} S_{q}(\widetilde{\omega})^{q /(q-2)}
$$

The formula follows.

We will use the following result in the proof of Theorem 1.1.

Lemma 2.2. Let $1<p<2^{*}$ and let $B_{r}$ and $A_{r}$ be as above with $0<r<1$. Then $\lim _{r \rightarrow 0} S_{p}^{\mathrm{rad}}\left(A_{r}\right)=S_{p}(B)$.

Proof. Let $u$ be a minimizer for $S_{p}(B)$. It is well known that $u$ is a radial function. Now let $v \in C^{\infty}(\mathbb{R})$ be such that $0 \leq v \leq 1, v(r)=0$ for $0 \leq r \leq 1$ and $v(r)=1$ for $r \geq 2$. Define $u_{n}(x)=v(n|x|) u(x)$ for $x \in B$. We have that $u_{n} \rightarrow u$ and $v_{n}(n|x|)\left(\partial u / \partial x_{k}\right) \rightarrow \partial u / \partial x_{k}$ in $L^{2}(B)$ for all $1 \leq k \leq N$. On the other hand by Hardy's inequality we have

$$
n u(x) v^{\prime}(n|x|)=\frac{u(x)}{|x|} n|x| v^{\prime}(n|x|) \leq 2\left|v^{\prime}\right|_{\infty} \frac{u(x)}{|x|} \in L^{2}(B),
$$

so that $n v^{\prime}(n|x|) u(x) \rightarrow 0$ in $L^{2}(B)$. It follows that $u_{n} \rightarrow u$ in $H_{0}^{1}(B)$. 
Now $S_{p}(B)<S_{p}^{\mathrm{rad}}\left(A_{r}\right)$ since $A_{r} \varsubsetneqq B$ for all $0<r<1$. On the other hand if $r=1 / n$ then

and

$$
S_{p}^{\mathrm{rad}}\left(A_{r}\right) \leq \frac{\int_{B}\left|\nabla u_{n}\right|^{2} d x}{\left(\int_{B} u_{n}^{p} d x\right)^{2 / p}}
$$

$$
\frac{\int_{B}\left|\nabla u_{n}\right|^{2} d x}{\left(\int_{B} u_{n}^{p} d x\right)^{2 / p}} \rightarrow S_{p}(B) \quad \text { as } n \rightarrow \infty .
$$

Proof of Theorem 1.1. Statement (1.1). Let $\lambda_{r}$ be the second radial eigenvalue of the Laplacian and let $B_{r_{0}}$ and $A_{r_{0}}$ be the nodal domains of an associated eigenfunction. Then it is well known that $\lambda_{r}=S_{2}\left(B_{r_{0}}\right)=S_{2}\left(A_{r_{0}}\right)$. Let $\lambda_{2}(B)$ be the second eigenvalue of the Laplacian. It is well known that the nodal domains of an associated eigenfunction are precisely two half balls so that $\lambda_{2}(B)=S_{2}\left(B^{+}\right)=S_{2}\left(B^{-}\right)$. Since $\lambda_{2}(B)<\lambda_{r}$ we have $S_{2}\left(B_{r_{0}}\right)>S_{2}\left(B^{+}\right)$and $S_{2}\left(A_{r_{0}}\right)>S_{2}\left(B^{-}\right)$. Let us consider first the inequality $S_{2}\left(B_{r_{0}}\right)>S_{2}\left(B^{+}\right)$. By continuity there is a number $\delta>0$ and $c>1$ such that $S_{p}\left(B_{r_{0}}\right)>c S_{p}\left(B^{+}\right)$for any $p \in[2,2+\delta]$. It follows that for some $\delta>0$ small enough we have

$$
\left.\left.\left(\frac{S_{p}\left(B_{r_{0}}\right)}{S_{p}\left(B^{+}\right)}\right)^{p /(p-2)}>2 \text { for all } p \in\right] 2,2+\delta\right] .
$$

Similarly,

$$
\left.\left.\left(\frac{S_{p}\left(A_{r_{0}}\right)}{S_{p}\left(B^{-}\right)}\right)^{p /(p-2)}>2 \text { for all } p \in\right] 2,2+\delta\right] .
$$

Using that $S_{2}\left(B^{+}\right)=S_{2}\left(B^{-}\right)$we then get

$$
\frac{p-2}{2 p} S_{p}\left(B_{r_{0}}\right)^{p /(p-2)}>\frac{p-2}{2 p} S_{p}\left(B^{+}\right)^{p /(p-2)}+\frac{p-2}{2 p} S_{p}\left(B^{-}\right)^{p /(p-2)},
$$

for all $p \in] 2,2+\delta]$. Now by continuity we have

$$
\frac{p-2}{2 p} S_{p}\left(B_{r_{0}}\right)^{p /(p-2)}>\frac{p-2}{2 p} S_{p}\left(B^{+}\right)^{p /(p-2)}+\frac{q-2}{2 q} S_{q}\left(B^{-}\right)^{q /(q-2)},
$$

for any $p \in] 2,2+\delta]$ and for any $q \in\left[p-\delta_{p}, p+\delta_{p}\right]$ for some $0<\delta_{p}<\delta$ that depends on $p$. Since $S_{p}\left(B_{r}\right)>S_{p}\left(B_{r_{0}}\right)$ when $r<r_{0}$, we have

$$
\begin{aligned}
\inf _{0<r<r_{0}} \frac{p-2}{2 p} S_{p}\left(B_{r}\right)^{p /(p-2)}+ & \frac{q-2}{2 q} S_{q}\left(A_{r}\right)^{q /(q-2)}>\frac{p-2}{2 p} S_{p}\left(B_{r_{0}}\right)^{p /(p-2)} \\
& >\frac{p-2}{2 p} S_{p}\left(B^{+}\right)^{p /(p-2)}+\frac{q-2}{2 q} S_{q}\left(B^{-}\right)^{q /(q-2)},
\end{aligned}
$$

for any $p \in] 2,2+\delta]$ and for any $q \in\left[p-\delta_{p}, p+\delta_{p}\right]$.

Using the fact that $S_{2}\left(A_{r}\right)>S_{2}\left(A_{r_{0}}\right)$ when $r>r_{0}$, we get

$$
\begin{aligned}
\inf _{r_{0}<r<1} \frac{p-2}{2 p} S_{p}\left(B_{r}\right)^{p /(p-2)}+ & \frac{q-2}{2 q} S_{q}\left(A_{r}\right)^{q /(q-2)} \\
& >\frac{p-2}{2 p} S_{p}\left(B^{+}\right)^{p /(p-2)}+\frac{q-2}{2 q} S_{q}\left(B^{-}\right)^{q /(q-2)},
\end{aligned}
$$


for any $p \in] 2,2+\delta]$ and for any $q \in\left[p-\delta_{p}, p+\delta_{p}\right]$. Combining the above inequalities we have

$$
\begin{aligned}
\inf _{0<r<1} \frac{p-2}{2 p} S_{p}\left(B_{r}\right)^{p /(p-2)} & +\frac{q-2}{2 q} S_{q}\left(A_{r}\right)^{q /(q-2)} \\
& >\frac{p-2}{2 p} S_{p}\left(B^{+}\right)^{p /(p-2)}+\frac{q-2}{2 q} S_{q}\left(B^{-}\right)^{q /(q-2)}=\beta_{h},
\end{aligned}
$$

for any $p \in] 2,2+\delta]$ and for any $q \in\left[p-\delta_{p}, p+\delta_{p}\right]$. Clearly we can swap $p$ and $q$ in the above inequality. This proves the first statement.

Statement (b). Let $p$ be fixed with $2<p<2^{*}$. We consider first the case when $q$ is near to 2 . There holds $S_{2}\left(B^{+}\right)>S_{2}(B)=\lambda_{1}(B)=j_{N / 2-1,1}^{2}$ where $\lambda_{1}(B)$ is the first eigenvalue of the Laplacian and $j_{N / 2-1,1}$ is the first zero of the Bessel function of the first kind and of order $N / 2-1$. By [3] we have that $S_{2}(B)>j_{0,1}^{2}+(N / 2-1)^{2}>1$. Thus by continuity there is a $\delta>0$ and $c>1$ such that

$$
S_{q}\left(B^{+}\right)>c S_{q}\left(B_{r}\right)>1
$$

for some fixed $r>0$ sufficiently near to 1 and for any $q \in[2,2+\delta]$. Now let

$$
\alpha=\left|\frac{p-2}{2 p} S_{p}^{\mathrm{rad}}\left(A_{r}\right)^{p /(p-2)}-\frac{p-2}{2 p} S_{p}\left(B^{-}\right)^{p /(p-2)}\right| .
$$

It follows from (2.6) that if $\delta>0$ is small enough then

$$
S_{q}\left(B^{+}\right)^{q /(q-2)}>S_{q}\left(B_{r}\right)^{q /(q-2)}+\alpha \frac{2 q}{q-2},
$$

for any $q \in] 2,2+\delta]$. Thus

$$
\begin{aligned}
\beta_{h}=\frac{q-2}{2 q} S_{q}\left(B^{+}\right)^{q /(q-2)} & +\frac{p-2}{2 p} S_{p}\left(B^{-}\right)^{p /(p-2)} \\
& >\frac{q-2}{2 q} S_{q}\left(B_{r}\right)^{q /(q-2)}+\frac{p-2}{2 p} S_{p}^{\mathrm{rad}}\left(A_{r}\right)^{p /(p-2)} \geq \beta_{\mathrm{rad}} .
\end{aligned}
$$

Now we consider the case when $q$ is near to $2^{*}$. Since $B^{+} \varsubsetneqq B$ we have $S_{p}\left(B^{+}\right)>S_{p}(B)$. By Lemma 2.2 there holds $\lim _{r \rightarrow 0} S_{p}^{\operatorname{rad}}\left(A_{r}\right)=S_{p}(B)$, thus we may fix $r>0$ small such that

$$
\frac{p-2}{2 p} S_{p}\left(B^{+}\right)^{p /(p-2)}>\frac{p-2}{2 p} S_{p}^{\mathrm{rad}}\left(A_{r}\right)^{p /(p-2)} .
$$

On the other hand

$$
\frac{q-2}{2 q} S_{q}\left(B_{r}\right)^{q /(q-2)} \rightarrow \frac{1}{N} S^{N / 2} \quad \text { and } \quad \frac{q-2}{2 q} S_{q}\left(B^{-}\right)^{q /(q-2)} \rightarrow \frac{1}{N} S^{N / 2},
$$


as $q \rightarrow 2^{*}$, where $S$ is the critical Sobolev constant. Consequently if $q$ is sufficiently near to $2^{*}$ then

$$
\begin{aligned}
\beta_{h}=\frac{p-2}{2 p} S_{p}\left(B^{+}\right)^{p /(p-2)}+\frac{q-2}{2 q} S_{q}\left(B^{-}\right)^{q /(q-2)} & \\
& >\frac{p-2}{p} S_{p}^{\mathrm{rad}}\left(A_{r}\right)^{2 p /(p-2)}+\frac{q-2}{2 q} S_{q}\left(B_{r}\right)^{q /(q-2)} \geq \beta_{\mathrm{rad}} .
\end{aligned}
$$

\section{The Neumann problem}

In this section we consider the problem

$$
\begin{cases}-\Delta u=u_{+}^{p-1}-u_{-}^{q-1} & \text { in } B \\ \frac{\partial u}{\partial \nu}=0 & \text { on } \partial B .\end{cases}
$$

Let the functional $\varphi$ given by (1.2) be defined over $H^{1}(B)$ and let

$$
\mathcal{H}=\left\{u \in H^{1}(B): u_{+} \neq 0, u_{-} \neq 0,\left\langle\varphi^{\prime}\left(u_{+}\right), u_{+}\right\rangle=\left\langle\varphi^{\prime}\left(u_{-}\right), u_{-}\right\rangle=0\right\} .
$$

As in the case of the Dirichlet boundary condition it can be shown that

$$
\eta=\inf _{u \in \mathcal{H}} \varphi(u)
$$

is achieved. Likewise, the procedure described in the proof of Proposition 3.1 of [2] carries over to the Neumann case with some obvious modifications and we get that any minimizer $u \in \mathcal{H}$ is a critical point of $\varphi$ having exactly two nodal domains. Such a function $u$ is called a least energy nodal solution of (3.1). As above we may define quantities $\eta_{\text {rad }}$ and $\eta_{h}$ as the infimum of $\varphi$ over the subsets of $\mathcal{H}$ consisting respectively of radial functions and of functions which are positive in $B^{+}$and negative in $B^{-}$. Let $\omega \subset B$ be a nonempty open subset and define

$$
\nu_{p}(\omega)=\inf _{u \in H_{*}^{1}(\omega)} \frac{\int_{\omega}|\nabla u|^{2} d x}{\left(\int_{\omega}|u|^{p} d x\right)^{2 / p}}
$$

where $H_{*}^{1}(\omega)$ is the subset of $H^{1}(B)$ consisting of functions which are zero in $B \backslash \bar{\omega}$. We have $\nu_{p}(B)=0$ whereas if $B \backslash \bar{\omega}$ is nonempty then there holds a Poincaré type embedding for functions in $H_{*}^{1}(\omega)$ and it follows that $\nu_{p}$ is achieved by some $u \in H_{*}^{1}(\omega)$ and that $\nu_{p}>0$. When $\omega$ is a radial domain we denote by $\nu_{p}^{\operatorname{rad}}(\omega)$ the infimum (3.3) taken over radial functions. Let us remark that, for $0<r<1$, $\nu_{p}\left(B_{r}\right)=S_{p}\left(B_{r}\right)$. As in Proposition 2.1 we have the following characterizations.

$$
\begin{aligned}
\eta_{h}= & \frac{p-2}{2 p} \nu_{p}\left(B^{+}\right)^{p /(p-2)}+\frac{q-2}{2 q} \nu_{q}\left(B^{-}\right)^{q /(q-2)}, \\
\eta_{\mathrm{rad}}= & \min _{0<r<1} \min \left\{\frac{p-2}{2 p} \nu_{p}\left(B_{r}\right)^{p /(p-2)}+\frac{q-2}{2 q} \nu_{q}^{\mathrm{rad}}\left(A_{r}\right)^{q /(q-2)} ;\right. \\
& \left.\frac{p-2}{2 p} \nu_{p}^{\mathrm{rad}}\left(A_{r}\right)^{p /(p-2)}+\frac{q-2}{2 q} \nu_{q}\left(B_{r}\right)^{q /(q-2)}\right\} .
\end{aligned}
$$


TheOREM 3.1. Let $\eta_{h}$ and $\eta_{\mathrm{rad}}$ be as above.

(a) If $p$ is sufficiently near to 2 and if $q$ is sufficiently near to $p$ then $\eta_{h}<$ $\eta_{\mathrm{rad}}$. In particular if $q=p$ is near to 2 then the conclusion holds.

(b) Let $p$ be fixed with $2<p<2^{*}$. If $q$ is sufficiently near to 2 then $\eta_{\mathrm{rad}}<\eta_{h}$.

A statement similar to (b) holds when $q$ is fixed and $p$ varies.

Proof. The proof of the first statement in Theorem 3.1 is similar to the proof of the first statement in Theorem 1.1 and we will not include it here. Let us consider the second statement. Using a similar argument to the one appearing in the proof of Lemma 2.2 one may show that $\lim _{r \rightarrow 0} \nu_{2}^{\mathrm{rad}}\left(A_{r}\right)=\nu_{2}(B)=0$. Thus we may fix $r>0$ small such that $\nu_{2}^{\mathrm{rad}}\left(A_{r}\right)<1$. On the other hand $\nu_{2}\left(B^{+}\right)$ is the first nonzero eigenvalue of the Laplacian with a homogeneous Neumann condition and it is well known that $\nu_{2}\left(B^{+}\right)=j_{N / 2,1}^{\prime}>1$, where $j_{N / 2,1}^{\prime}$ is the first zero of the derivative of the Bessel function of the first kind and of order $N / 2$. Now, by continuity, there is $0<\varepsilon<1$ and $\delta>0$ such that

$$
\nu_{q}^{\mathrm{rad}}\left(A_{r}\right)<1-\varepsilon \text { and } \nu_{q}\left(B^{+}\right)>1+\varepsilon,
$$

for any $q \in[2,2+\delta]$. Now let $2<p<\infty$ be fixed and let

$$
\alpha=\left|\frac{p-2}{2 p} \nu_{p}\left(B_{r}\right)^{p /(p-2)}-\frac{p-2}{2 p} \nu_{p}\left(B^{-}\right)^{p /(p-2)}\right| .
$$

From (3.6) we may choose $\delta>0$ small such that

$$
\nu_{q}\left(B^{+}\right)^{q /(q-2)}>\nu_{q}^{\mathrm{rad}}\left(A_{r}\right)^{q /(q-2)}+\frac{2 q}{q-2} \alpha,
$$

for any $q \in] 2,2+\delta]$. We then obtain $\eta_{h}>\eta_{\text {rad }}$ as in the proof of Theorem 1.1.

\section{Numerical computations}

In what follows we assume $q=p$. By Theorem 1.1 we have $\beta_{h}<\beta_{\text {rad }}$ provided $p$ is sufficiently near to 2 . It has been suggested that in fact $\beta_{h}<\beta_{\text {rad }}$ for any $2<p<2^{*}$. We have tested the validity of this statement numerically for $N=3$.

First we obtain an approximation of $\beta_{\text {rad }}$. Let $a>0$ and let $u_{a}$ be a solution of the equation

$$
-u^{\prime \prime}(r)=\frac{N-1}{r} u^{\prime}(r)+|u(r)|^{p-2} u(r)
$$

on $] 0,1\left[\right.$ with Cauchy data $u(0)=a$ and $u^{\prime}(0)=0$. We may adjust $a$ in such a way that $u_{a}$ has exactly two nodal domains and $u_{a}(1)=0$. Thus if $v$ denotes a minimizer for $\beta_{\text {rad }}$ then $u_{a}$ and $v$ are two radial solutions of the boundary value problem (1.1) having exactly two nodal domains. By the uniqueness result of [4] we have $u_{a} \equiv v$. Thus we may compute $\beta_{\text {rad }}=\varphi\left(u_{a}\right)$. 
An upper bound for $\beta_{h}$ is given by $\widetilde{\beta}=\varphi(f)$ for some $f \in \mathcal{M}_{h}$. When $2<p \leq 4.5$ we take $f$ to be a second eigenfunction of the Laplacian. When $4.5<p<2^{*}=6$ let $\lambda>0$ and let $v(|x|)=\left(\lambda+|x|^{2}\right)^{-1 / 2}-(\lambda+1 / 4)^{-1 / 2}$ for $0<|x|<1 / 2$ and $v(|x|)=0$ for $|x|>1 / 2$. We may translate $v$ so that its support lies in $B^{+}$and then take $f$ to be an appropriate multiple of the odd extension of $v$ to $B$. Table 1 contains data which suggests that $\beta_{h}<\widetilde{\beta}<\beta_{\text {rad }}$ for a large range of values of $p$. To compute the last three columns for $\widetilde{\beta}$ we took respectively $\lambda=10^{-2}, 10^{-3}$ and $10^{-5}$.

\begin{tabular}{|c|c|c|c|c|c|c|}
\hline$p$ & 2.5 & 3.5 & 4.5 & 5.5 & 5.7 & 5.9 \\
\hline$\beta_{\mathrm{rad}}$ & $1.2 \mathrm{e} 7$ & 912.4 & 96.54 & 21.97 & 15.98 & 11.03 \\
\hline$\widetilde{\beta}$ & $5.1 \mathrm{e} 5$ & 313.2 & 73.63 & 17.61 & 12.61 & 9.898 \\
\hline
\end{tabular}

TABLE 1. $\beta_{\text {rad }}$ and $\widetilde{\beta}$ with $N=3$.

Acknowledgements. The author extends his warmest thanks to Pr. Michel Willem for suggesting the problem considered in this paper and for providing various insightful comments including Lemma 2.2 and its proof.

\section{REFERENCES}

[1] T. BARTsCh And T. Weth, A note on additional properties of sign changing solutions to superlinear elliptic equations, Topol. Methods Nonlinear Anal. 22 (2003), 1-14.

[2] T. Bartsch, T. Weth And M. Willem, Partial symmetry of least energy nodal solutions to some variational problems, J. Anal. Math. (to appear).

[3] R. McCann, Lower bounds for the zeros of Bessel functions, Proc. Amer. Math. Soc. 64 (1977), 101-103.

[4] K. NAgasaki, Radial solutions for $\Delta u+|x|^{l}|u|^{p-1} u=0$ on the unit ball in $\mathbb{R}^{n}$, J. Fac. Sci. Univ. Tokyo Sect. IA Math. 36 (1989), 211-232.

Olaf TORnÉ

Université Libre de Bruxelles

Campus de la Plaine, ULB CP214

Boulevard du Triomphe

1050 Bruxelles, BELGIUM

E-mail address: otorne@ulb.ac.be 